\section{A Premier Event}

\section{G. Chiarotti who chaired the International Programme Committee of the 15th General Conference of the Condensed Matter Division of EPS (Baveno-Stresa; 22-25 April 1996), reports that the conference has established itself as one of the premier events in its field.}

To point out the highlights of a conference is always a difficult task. Doubtless one makes more enemies than friends ! Nevertheless I shall try to summarise my general impressions of 15 th General Conference of the Condensed Matter Division of EPS which took place beside Lake Maggiore last April. The remarks are biased of course by my own scientific interests and tastes.

Some of the more exciting results discussed at the conference dealt with BoseEinstein condensation in (extremely) cold alkali vapours, a phenomenon that was discovered only a few months ago. In a plenary invited talk, W. Ketterle, who works on the team that made the first observation in sodium at MIT, described the fantastic cocktail of experimental techniques (extremely low temperatures, laser imaging, etc.) that led to the observation of a condensate of as many as $5 \times 10^{5}$ atoms [see EN 27 (1996) 105]. The conference was also able to offer an invited talk on the theory of Bose-Einstein condensation in

magnetically trapped atomic gases by $\mathrm{S}$. Stringari, and an contribution by P. Wachter on the excitonic insulator in rare-earth compounds, a system that is believed to undergo Bose-Einstein condensation.

Computational physics, especially molecular dynamics and Car-Parrinello $a b$-initio simulations, was a subject that undoubtedly received great attention. Once limited to special problems in solid and liquid dynamics, it has now pervaded almost every field of condensed matter. Computational results were presented for matter under extreme conditions, especially high pressures; diffusion; soft matter; etc. The conference offered a total of eight invited talks and eight oral contributions on computational physics. They were complemented by a plenary lecture by $\mathrm{E}$. Tosatti on order-disorder transitions at surfaces, which although based primarily on physical concepts and intuition often relied on a $b$ initio calculations. Another plenary talk in the special session on
Speakers at the CMD'96 Special Plenary Session on Applied Physics that was chaired by G. Chiarotti, the Chairman of the CMD'96 International Programme Committee. From the left, F. Carpasso (AT\&T Bell Laboratories, NY, USA), B. Smit (Shell Research, Amsterdam) and O.V. Lounesmaa (Helsinki).
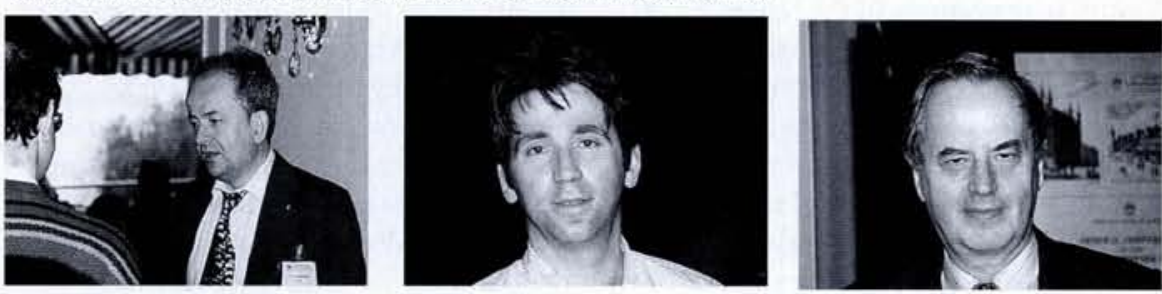

Industrial exhibitors are an important feature of all major conferences that seek to promote the application of physics research. Some 25 companies exhibited at CMD'96, among them Magnetic Solutions, Ltd. a start-up founded in 1994 by academic and research staff from Trinity College, Dublin (TCD). The company, which is based at the TCD Innovation Centre, designs and manufactures advanced magnet systems, some of which are sold under the slogan "Forget Electromagnets". To understand why, please read the article on page 186 by J.M.D. Coey, the company's Managing Director, who spoke during the CMD'96 symposium. He is photographed here with David Hurley, the company's Manager, on the left. The Centre aims to start up 2-3 new companies each year in areas in which the university can contribute and become involved; they then usually move out after about three years.

Magnetic Solutions is unusual in that it has started manufacturing within a comparatively short time. One innovative product is a compact pneumatically driven permanent magnet flux source based on an original system of split-pair Halbach cylinder permanent magnets (page 188). The source shown in the photograph generates large, switchable magnetic fields which are ideal for synchrotron beamline experiments. applied physics - by B. Smit on the catalytic cracking of oil molecules (see page 189) - also spoke in detail about the results of simulations. Some of the problems are indeed formidable so physical intuition (fortunately !) still plays a crucial role. Paradigmatic in this respect was the title Physical Chemistry with Computers and Intuitions of the invited talk by M.L. Klein.

The session Semiconductors and Insulators was by far the largest as it covered several related symposia and more than 250 submitted contributions. A great deal of research in this field is devoted to nanostructured materials (including quantum wells, wires and dots, clusters and fullerenes, carbon nanotubes and microactivities, etc.). An interesting trend, clearly noticeable at the conference, was that these techniques, once limited to semiconductors, are now spreading to other fields such as superconductors (antidot lattices; artificial multilayers for high- $T_{c}$ superconductivity), magnetic materials, assisted diffusion, etc., where nanoscale fabrication opens up unexpected possibilities. A plenary invited talk New Phenomena in Nanostructured Materials by Y. Bruynseraede surveyed applications in superconductors and metals.

Reduction of length scales allows the observation of quantum interference effects and their use in electronic devices. We are all well acquainted with band-gap engineering in semiconductors. F. Capasso in his plenary lecture showed that wavefunction engineering is also possible through the control of layer thickness in multilayer structures. Taking advantage of the structure of the wavefunction, it is now possible to control the transition probability of electrons across the barriers,

increasing or suppressing emission at certain energies. Through such mechanisms, quantum cascade lasers that emit as much as $100 \mathrm{~mW}$ in the infrared $(5-10 \mu \mathrm{m})$ have been built.

A result of fundamental research, and one that can be traced to the same type of problem, is the observation of coherent dynamics of a single-electron wavepacket in $\mathrm{KBr}$ using femtosecond laser techniques. The authors (R. Scholz et al.; contributed paper) were able to follow the time evolution of the wave packet of the excited electron in the F-center, observing damped periodic oscillations of the sample transmission in the Thz range. The configurational coordinate model (once inferred from the Stokes shifts in luminescence) can now be checked directly by observing the vibronic frequencies. 
Another result that depends in a dramatic way on improvements in experimental techniques was presented by F. Sette in a plenary lecture. The availability at the European Synchrotron Radiation Facility in Grenoble of intense and collimated beams of X-rays allowed him to reach energy resolutions of $3.2 \mathrm{meV}$ with beams of $10-20 \mathrm{keV}$. As an example, he discussed experimental results on the dispersion of longitudinal acoustic excitations in water, in a range of momentum transfer that corresponds to propagation velocities twice the speed of sound in the hydrodynamic limit [see EN 26 (1995) 78].

One last improvement in experimental techniques that is worth mentioning is the possibility - achieved recently using Xrays from synchrotron radiation sources of surface-structure determinations. Such methods, which were discussed in an invited talk by R.L. Johnson on semiconductor surfaces studied with STM and Xray diffraction, successfully complement other surface-sensitive techniques.

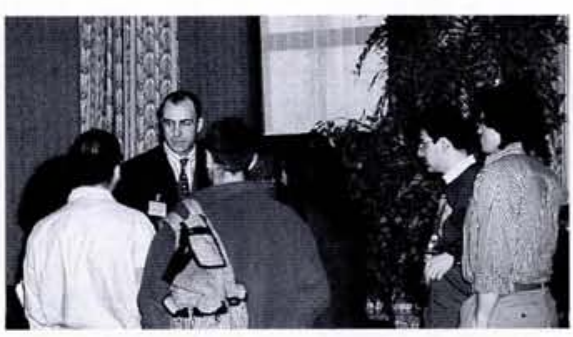

Stimulating employment opportunities for young physicists has become a major preoccupation. One approach is the setting up of some form of "job market" at large general conferences which have a bias towards applications. It has been very successful in the United States, where the American Physical Society's March meeting provides students and young physicists with detailed information about job openings. Offering this service has the additional advantage that young people are encouraged to attend major scientific meetings.

The same approach is poorly developed in the much less homogeneous European context. So the CMD'96 organizers made a determined effort to provide job information and counselling. This was a success and they were able to establish job placement as an integral feature of the CMD conference series (CMD'97 takes place in Leuven on 25 - 28 July 1997). Initiatives were based on a Placement Centre where young people registered their details and received announcements from

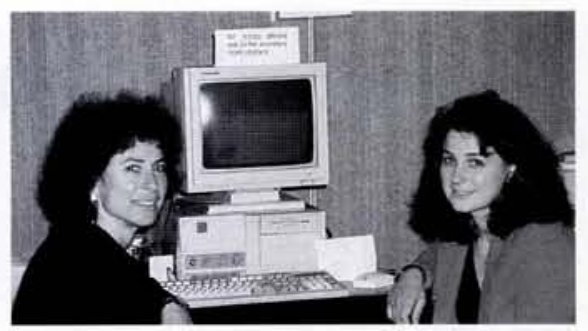

potential employers. The Centre also hosted a series of well-attended presentations by laboratory directors and government officials, including Marco Malacarne from the European Commission's science directorate who is seen here answering questions on fellowship opportunities within European Union programmes. Another feature was an introduction to the Sportello Giovani online interactive WWW database of candidates seeking employment and job opportunities. The service is clearly a pioneer in using the Web to provide job information. Hosted in Genoa by the Istituto Nazionale per la Fisica della Materia (INFM) with support from the EC as an accompanying measure, it is administered by Matilde Bolla (seen on the right in the photograph with Manuela Arata, the INFN's Director General). Sportello Giovani (http://www.infm.it) currently details 200 job offers ( $80 \%$ in Europe) based essentially on published advertisements and information from Italian research agencies (INFN, INFM, CNR) and universities.

\section{5th General Conference of the Condensed Matter Division}

More than 800 scientists from 37 countries attended the 15th General Conference of the EPS Condensed Matter Division. Though the commendable efforts of the Organizing Committee and the support of some national and international agencies, a large participation (more than 100 scientists). from east and central Europe was possible. Participants from outside Europe amounted to 29 .

The conference clearly established itself as a premier venue for the worldwide exchange of scientific and results in the field of condensed matter, in a way comparable to the American Physical Society's March meeting.

The conference consisted of six main sessions (Semiconductors and Insulators, Magnetism and Metals, Superconductivity, Liquids and Statistical Mechanics, Polymers and Soft Matter, Surfaces and Interfaces) and 14 symposia dealing with some of the leading fields of present research. The symposia elucidate the scientific trends covered by the conference as planned by the International Programme Committee so it is useful to recall their titles:

- High-pressure phases of low- $Z$ elements

- Isotopically engineered materials

- Computational physics in condensed matter

- Clusters and fullerenes

- Point-contact spectroscopy

- Scanning tunnelling microscopy

- High-Tc superconductors

- Vortices in superconductors and superfluids

- Magnetic nanostructures

- Observation of surfaces at atomic resolution

- Atomic mobility and tunnelling

- Semiconductor nanostructures and cavities

- Conducting polymers

- Thin polymer layers and interfaces.
The conference offered a relatively large number of plenary invited talks (12, including the lecture by R.H. Friend, the winner of the 1996 Hewlett-Packard Europhysics Prize) and invited talks (69 in parallel sessions). Some 180 contributions papers were presented orally and 660 as posters.

The Programme Committee aimed to stress the importance of condensed matter research in the development of our society by organizing two special events. The first was a full morning plenary session devoted to applied solid-state physics with invited talks by J.M.D. Coey on Industrial applications of permanent magnets, F. Capasso on Quantum cascade lasers, E. Smit on Computational physics in industry, O.V. Lounasmaa on Medical applications of SQUIDS in neuro- and cardiomagnetism, and R.H. Friend on Semiconductors device physics with conjugated polymers. There was also an evening plenary session devoted to Science and Society with an invited talk Past, present and future of industrial research in solid-state physics by J.C. Phillips [see EN 27 (1996) 125] followed by an impromptu discussion, coordinated by $\mathrm{N}$. Kroó, on European Union programmes.

A forum on (proposed) new European large facilities (far-infrared free-electron lasers, physics in the megagauss region, and cold neutron sources) with three invited speakers (K. Unterrainer, M. Springford, A. Furrer, respectively) was organized as a special parallel session.

Other plenary invited lectures were by: R.A. Cowley on Are there two lengths scales at a phase transition?; W. Ketterle on Observation of Bose-Einstein condensation in ultra-

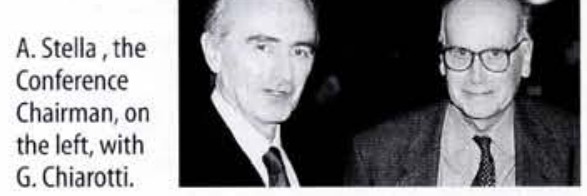

cold atomic gases; E. Tossatti on Order and disorder at crystal surfaces; T.P. Martin on $C_{60}$ molecules with a metallic outer layer; F. Sette on Collective dynamics in liquid water by inelastic X-ray scattering with meV resolution; $\mathrm{Y}$. Bruynseraede on New phenomena in nanostructured materials.

Mention should be made of the Placement Centre (see above) and twelve "young author awards" for the best contributions presented by young scientists (under 30 years of age) were offered by the Istituto Nazionale per la Fisica della Materia.

The conference was a great success. Attendance at the sessions (including the closing session) was very high and the discussions were always lively and extended. And the weather remained acceptable for most of the time ! Moreover, the participation of young scientists exceeded expectations. The people who made this success possible were A. Stella (Conference Chairman), I.C. Andreani (Scientific Secretary), G. Guizzetti (Chairman of the Local Committee), and the members of the International Programme Committee, namely V.M. Agranovich, M. Altarelli, F.J. Balta-

Calleja, F. Bassani, R. Cantelli, M. Cardona, G. Chiarotti (chair), F, Cyrot-Lackman, J.T. Devreese, J.E. Enderby, F. Flores, P. Fulde, V.V. Mishchalkov, R.J. Nocholas, M. Parrinello, E. Rimini, M.P. Tosi, P. Wachter, K. Wandelt, and P. Wyder. 